\title{
Metabolic disorders in menopause
}

\author{
Grzegorz Stachowiak ${ }^{1}$, Tomasz Pertyński², Magdalena Pertyńska-Marczewska ${ }^{3}$ \\ ${ }^{1}$ Department of Gynecology and Gynecological Oncology, Polish Mother's Memorial Hospital-Research Institute, Lodz, Poland \\ ${ }^{2}$ M. Kopernik County Specialist Hospital, Lodz, Poland \\ ${ }^{3}$ Independent Consultant Obstetrics and Gynecology, London, United Kingdom
}

\begin{abstract}
Metabolic disorders occurring in menopause, including dyslipidemia, disorders of carbohydrate metabolism (impaired glucose tolerance - IGT, type 2 diabetes mellitus - T2DM) or components of metabolic syndrome, constitute risk factors for cardiovascular disease in women. A key role could be played here by hyperinsulinemia, insulin resistance and visceral obesity, all contributing to dyslipidemia, oxidative stress, inflammation, alter coagulation and atherosclerosis observed during the menopausal period. Undiagnosed and untreated, metabolic disorders may adversely affect the length and quality of women's life. Prevention and treatment preceded by early diagnosis should be the main goal for the physicians involved in menopausal care. This article represents a short review of the current knowledge concerning metabolic disorders (e.g. obesity, polycystic ovary syndrome or thyroid diseases) in menopause, including the role of a tailored menopausal hormone therapy (HT). According to current data, HT is not recommend as a preventive strategy for metabolic disorders in menopause. Nevertheless, as part of a comprehensive strategy to prevent chronic diseases after menopause, menopausal hormone therapy, particularly estrogen therapy may be considered (after balancing benefits/risks and excluding women with absolute contraindications to this therapy). Life-style modifications, with moderate physical activity and healthy diet at the forefront, should be still the first choice recommendation for all patients with menopausal metabolic abnormalities.
\end{abstract}

Key words: menopause, insulin resistance, diabetes, metabolic syndrome, PCOS.

\section{Introduction}

Beginning in the sixth decade of life, many chronic diseases begin to emerge, affecting both the quality and quantity of a woman's life. Depletion of sex steroid hormones is an important consequence of normal ageing and gonadal failure potentially increasing vulnerability to disease in hormone-responsive tissues, including the brain, bone and the cardiovascular system [1]. When women progress to hormonal menopause (follicle-stimulating hormone $[\mathrm{FSH}]>30 \mathrm{IU} / \mathrm{l}$, estradiol $\left[\mathrm{E}_{2}\right]<30 \mathrm{pg} /$ $\mathrm{ml}$, FSH/luteinizing hormone $[\mathrm{LH}]>1$ ), primarily in the state of absolute hypoestrogenism and subject to progressive ageing, the most characteristic complaints in menopause include vasomotor symptoms such as hot flashes and night sweats, urogenital atrophy, osteopenia and osteoporosis, psychiatric disorders, sexual dysfunction, skin lesions, cardiovascular disease, cancer, and finally, metabolic disorders and obesity. All of these adversely affect women's quality of life [1, 2].

The most common metabolic disorders in menopause include dyslipidemia, impaired glucose tolerance, insulin resistance, hyperinsulinemia, and type 2 diabetes (T2DM).

\section{Lipid metabolism disorders}

The decline in estradiol following the menopausal transition leaves the vasculature vulnerable to cardiovascular disease (CVD) risk factors, such as lipids [3]. Dyslipidemia in menopause is characterized by an increase in low-density lipoprotein (LDL) levels and a decline in high-density lipoprotein (HDL) levels [4]. In the Healthy Women Study, total and LDL cholesterol increased and $\mathrm{HDL}$ and $\mathrm{HDL}$, cholesterol declined among premenopausal women who had ceased menstruating for at least 1 year compared to age-matched premenopausal women who continued menstruating [5]. Interestingly, both the Los Angeles Atherosclerosis Study and the SWAN Heart Women demonstrated that the antiatherogenic effect of HDL diminishes in women around the age of menopause [6] and it was suggested that it is possibly related to changes in the lipoprotein subclass profile observed during the menopausal transition [7]. These changes have a clear negative impact on the cardiovascular system, accelerating the development of atherosclerosis.

In addition, Yazdani et al. demonstrated that in 31 women within 6 months from hysterectomy and bilat-

Corresponding author: Grzegorz Stachowiak, Department of Gynecology and Gynecological Oncology, Polish Mother's Memorial Hospital-Research Institute, 281/289 Rzgowska St., 93-338 Lodz, Poland, e-mail: gstach23@interia.pl

To cite this article: Stachowiak G, Pertyński T, Pertyńska-Marczewska M. Metabolic disorders in menopause. Prz Menopauzalny 2015; $14: 59-64$. Submitted: 7.09.2014; Accepted: 14.01.2015. 
eral salpingo-oophorectomy, triglycerides (TG), total cholesterol, and LDL had significantly increased and HDL slightly increased but without any significant difference [8]. The study by Kabir et al. compared 30 women with normal and surgical menopause and showed that TG was higher and the LDL cholesterol was lower in the surgical group [9]. A similar study was performed by Tuna et al. in two groups of 50 subjects each, demonstrating lower HDL and higher very low density lipoprotein (VLDL) cholesterols in the surgical group [10].

It is widely recognized that the use of hormone therapy $(\mathrm{HT})$ has a beneficial effect on lipids, reversing the changes mentioned above. This was confirmed by this year's meta-analysis (comprising 16 randomized, placebo-controlled research studies conducted in 19972011; 17971 cases were included), demonstrating that combined estrogen replacement therapy (ERT) dramatically reduced plasma total cholesterol and LDL. However, there was a slight increase in HDL levels as compared with placebo control [11].

\section{Disorders of carbohydrate metabolism}

Menopause should represent a period of increased 'diabetes vigilance' as a number of common symptoms reported by women in menopause such as a change in body weight (a significant increase or loss), polyuria, recurrent urogenital inflammation, fatigue, weakness, irritability, blurred vision, thirst, increased appetite, sexual dysfunction could point not to climacteric syndromes, but to carbohydrate disorder in the form of non-insulin-dependent T2DM [12].

Some report suggest that in some women, the estrogen deficiency that occurs after menopause could represent a fundamental step in the process of diabetogenesis [13]. Furthermore, ageing per se is associated with an increased risk of non-insulin-dependent T2DM [14]. Other risk factors for T2DM in menopause include obesity, low physical activity, poor diet, smoking, alcohol (in excess) and certain medications. Furthermore, the impaired metabolism of vitamin $D_{3}$ and calcium deficiency (distinctive in postmenopause) translate into an increased risk of both types of diabetes - type 1 diabetes mellitus (T1DM) and T2DM [15].

In postmenopausal women, endogenous bioavailable testosterone has been positively associated, and sex hormone-binding globulin (SHBG) has been negatively associated, with incident T2DM. Furthermore, circulating SHBG concentrations are inversely correlated with insulin resistance, and according to the Women's Health Study, a low level of SHBG is a strong predictor of T2DM in women $[16,17]$.

Although no HT product has a regulatory agency's approval to prevent DM, large randomized controlled trials (RCTs) demonstrate that HT reduces the new onset of T2DM.
The Heart and Estrogen/progestin Replacement Study (HERS) and Women's Health Initiative (WHI) studies showed a $35 \%$ and $21 \%$, respectively, reduction in the incidence of T2DM during HT [18, 19]. This observation provides important insights into the metabolic effects of postmenopausal hormones. These results are supported by a new meta-analysis, which demonstrated that women with combined ERT have significantly lower levels of fasting plasma glucose and hemoglobin $\mathrm{A}_{1 \mathrm{c}}\left(\mathrm{HbA}_{1 \mathrm{c}}\right)$ compared with placebo [11].

\section{Polycystic ovary syndrome and postmenopause}

The available research suggests that hyperandrogenism featuring women with polycystic ovary syndrome (PCOS) during their reproductive life persists also after menopause. Enhanced androgen production, assessed by total $\mathrm{T}$ concentrations, persists in postmenopausal women with ultrasonographic evidence of polycystic ovaries alone [20]. Adrenal androgens in PCOS remain high until menopause, whereas adrenal hyperandrogenism is not well documented in postmenopausal women with PCOS [21].

Postmenopausal women with PCOS have a higher free androgen index but lower FSH and SHBG than controls. Schmidt et al. reported also that menopausal age, body weight, body mass index (BMI), waist to hip ratio, $\mathrm{LH}$, prolactin, androstenedione, dehydroepiandrosterone sulfate, total testosterone, estradiol, and estrone were similar in PCOS and controls. Interestingly, women with PCOS reported hirsutism more frequently but had fewer climacteric symptoms and hypothyroidism than controls [22].

Furthermore, in another cross-sectional, universitybased hospital study by Puurunen et al., it was found that impaired glucose tolerance, enhanced ovarian androgen secretion and chronic inflammation observed in premenopausal women with PCOS persists after the menopausal transition [23].

Markopoulos et al. demonstrated that early postmenopausal PCOS women are characterized by hyperinsulinemia but with attenuated insulin resistance. Polycystic ovary syndrome women also had higher high-sensitivity C-reactive protein (hs-CRP), androgen and free androgen indices (FAI) levels than controls. Interestingly, in this study PCOS status and waist circumference were predictors of hyperinsulinemia while insulin sensitivity correlated negatively with FAI. The differences reported in adipocytokine levels between PCOS and non-PCOS women in reproductive years seem to disappear after menopause [24]. The observed insulin resistance could account for adverse health effects, such as diabetes and hypertension.

The prevalence of impaired glucose tolerance (IGT) and/or of T2DM in women with PCOS is estimated at 
around 40\% [25]. Danish researchers argue that the concentration of glycated hemoglobin $\left(\mathrm{HbA}_{1 \mathrm{c}} \geq 6.5 \%\right)$ may be a better marker of the cardiovascular risk than the glucose level in patients with PCOS [26].

Obesity, an inherent element of the Stein-Leventhal syndrome is observed in $50-60 \%$ of women with PCOS [27].

It is assumed that obesity, particular of visceral origin, insulin resistance (and T2DM), and persisting high androgen levels through the menopause, obesity and maturity onset diabetes mellitus in postmenopausal PCOS patients are proposed as the main mechanisms accounting for an unfavorable metabolic profile possessing at increased risk for cardiovascular events later in life. In addition to CVD risk factors, PCOS is also associated with early subclinical arterial disease. Even young women with PCOS have already increased intima-media thickness (IMT), evidence of arterial stiffness and signs of endothelial dysfunction [28].

At menopause, a woman with PCOS is likely to have had multiple cardiac risk factors for several decades and therefore is at risk of developing heart diseases. Thus, postmenopausal women with a history of PCOS, especially those with established diabetes and/or dyslipidemia, should be considered at high risk of the development of clinical cardiac diseases and therefore at increased risk of death later in life [29].

However, a prospective Swedish study demonstrated that although women with PCOS have an increased prevalence of hypertension and higher triglyceride levels, the incidence of myocardial infarction, stroke, diabetes, cancer and mortality prevalence was similar in the two cohorts with similar BMI. Therefore, the welldescribed cardiovascular/metabolic risk profile in preand perimenopausal PCOS women does not entail an evident increase in cardiovascular events during the postmenopausal period [30].

Metabolic syndrome (MetS) and PCOS appear to be interrelated, although they are distinct entities. Women with PCOS appear to be commonly affected by MetS, while women with MetS may display reproductive or endocrine features of PCOS. These clinical observations appear to be only partly attributable to the association of both syndromes with obesity and imply a reciprocal pathophysiologic relationship between PCOS and MetS leading to potentially significant clinical consequences $[31,32]$.

The presence of PCOS entangles the natural course of menopause, negatively affecting women's quality of life (quality of life - QoL). This is confirmed by reports of the working ESHRE/ASRM group, which demonstrated that women with PCOS are an at-risk group for psychological and behavioral disorders and reduced QoL, including an increased risk of mental disorders (anxiety, depression), eating disorders or sexual dysfunction [33].

\section{Obesity}

After menopause the incidence of obesity increases, including visceral (android) obesity. Such fat distribution fosters the occurrence of a number of metabolic disorders, including fully manifested metabolic syndrome, and enhances the development of atherosclerosis. Unfortunately, in Poland up to 2/3 of menopausal women are overweight or obese [34].

Toth et al. reported that postmenopausal women had greater amounts of whole body and intra-abdominal fat compared with premenopausal women. Plasma glycerol levels remained similar after statistical adjustment for fat mass, fat percentage, and intra-abdominal fat. Moreover, no differences in plasma glycerol were observed in pre- and postmenopausal women matched for fat mass or intra-abdominal fat. Their results suggested no effect of the menopausal status on plasma glycerol levels under postabsorptive or hyperinsulinemic conditions [35].

The comparison of patients with normal $\mathrm{BMI}$ and those with high BMI showed that high BMI (> $\left.30 \mathrm{~kg} / \mathrm{m}^{2}\right)$ had a significant negative effect on blood pressure (as evidenced by the increased frequency of hypertension in overweight and obese patients) that it also negatively and significantly affected triglyceride and fasting glucose levels, and that it was linked significantly to low levels of HDL-C, therefore with CVD risk factors [36].

Intra-abdominal fat cells produce a number of substances with an impact on inflammatory responses, insulin resistance and an increased risk of cardiovascular diseases. Some molecules, directly synthesized by adipocytes are called "adipokines". These include adipocytokines, such as leptin, adiponectin, resistin, ghrelin. They control energy balance and appetite, and influence insulin sensitivity via endocrine mechanisms, they also modulate adipocyte size/number and angiogenesis via paracrine mechanisms, thus exerting a major role in the regulation of fat mass [37]. Furthermore, they can also exert a role in the control of blood pressure, lipoprotein metabolism, coagulation, immunity and inflammation [38, 39].

Menopausal women are characterized by elevated levels of leptin and resistin and decreased levels of adiponectin and ghrelin ('high' leptin together with 'low' adiponectin show a positive correlation with insulin resistance markers). In postmenopause, excess fat will also result in an increased production of endogenous estrogen, resulting from occurring in situ extra glandular aromatization (androstenedione see: estrone) [40].

\section{Metabolic syndrome}

The prevalence of MetS differs greatly in different populations. Amongst pre-and postmenopausal women it ranges from $13.8 \%$ to more than $60.0 \%$ [41]. Weight 
gain and obesity largely drive the increased prevalence of MetS in postmenopausal women [42]. Menopausal transition is associated with significant weight gain ( 2 to $2.5 \mathrm{~kg}$ over 3 years on average).

Concomitantly, there is an increase in abdominal adiposity and a decrease in energy expenditure [43]. Thus, such phenomena have been postulated to explain the higher risk of metabolic syndrome and increases in cholesterol and triglycerides and therefore menopause is considered a predictor of MetS, independent of women's age [44]. Other risk factors for MetS include menarche age. Menarche age showed a 'U' shaped relationship with MetS and oligomenorrhea in adulthood. Late menarche and early menarche are risk factors for adult oligomenorrhea, MetS, and cardiometabolic abnormalities. Therefore, girls with early ( $\leq 10$ age) and with late menarche $(\geq 16)$ represent a group at high risk of adult cardiometabolic abnormalities and oligomenorrhea [45]. Also, in another study, irregular menses had been correlated with elevated levels of triglycerides and obesity [46].

Hyperinsulinemia, insulin resistance and (particularly visceral adiposity) obesity are the main features of MetS, found in many postmenopausal women. The simultaneous occurrence of insulin resistance and obesity is the most detrimental for metabolic health, and is also associated with increased oxidative stress, inflammatory and prothrombotic processes as well as with postmenopausal alterations in adipocytokine production [47].

In a cross-sectional study of postmenopausal women, the mean of age, BMI, weight and the mean levels of testosterone were higher and SHBG was lower in women with MetS. The correlation between SHBG and testosterone among women was particularly strong [48].

Chedraui et al. demonstrated that MetS women displayed significantly higher levels of adipsin, leptin, resistin, insulin and homeostasis model assessmentestimated insulin resistance (HOMA-IR) values and lower adiponectin levels. These differences were mainly observed among women with abdominal obesity, independent of fulfilling MetS criteria or not. In this same sense, lower adiponectin levels significantly related to low HDL-C and high triglyceride levels; and higher insulin and HOMA-IR values related to high triglyceride and glucose levels, respectively [49]. In another paper, Chedraui et al. reported that postmenopausal women with MetS showed higher interleukin 6 (IL-6) (inflammation) and lower urokinase-type plasminogen activator (UPA) levels (endothelial dysfunction). Moreover, IL-6 levels were higher among women with abdominal obesity, low HDL-C and high triglyceride levels. Women with low HDL-C and high triglyceride levels presented significantly lower uPA levels. These were mainly related to metabolic and lipid abnormalities [50].

Female sexual dysfunction is a prevalent and multidimensional disorder related to many biological, psy- chological, and social determinants. It was also shown that the incidence of MetS in pre- and postmenopausal women adversely affects their sexuality. The authors of the study used the Female Sexual Function Index to assess women's sexual function. Female sexual dysfunction was found more often in both pre- and postmenopausal women with metabolic syndrome. The Overall Female Sexual Function Index score and satisfaction, pain, and desire domain scores independently of the menopause status showed statistically significant differences across women with metabolic syndrome in comparison to participants with no metabolic syndrome. This study clearly showed that sexual dysfunction is more prevalent in pre- and postmenopausal women with metabolic syndrome [51].

The use of HT is beneficial overall for reducing many of the parameters of MetS. According to Lobo, in MetS, transdermal therapy may be preferable to oral therapy, when given in standard doses. Women with MetS have elevations in several inflammation and coagulation factors. Both oral and transdermal HT reduce inflammation markers except for levels of CRP and matrix metallopeptidase 9 (MMP-9), which increase with oral therapy, but are unaffected by the transdermal route. The beneficial effects of HT on lipids occur in postmenopausal women with and without MetS, although the changes in the latter were minimal. Blood pressure was not affected by HT in women with MetS [42].

Furthermore, the use of oral estrogen therapy (ET) in a group of postmenopausal women with MetS increases the levels of leptin and resistin and decreases the levels of ghrelin. Transdermal ET increases the levels of adiponectin and decreases the levels of ghrelin. This confirms the more beneficial effect of the transdermal route (compared to the oral route), on the MetS parameters in postmenopausal women [52].

\section{Thyroid diseases}

The relatively few studies on the relationship between menopause and thyroid functions do not allow to clarify whether menopause has an effect on the thyroid regardless of the ageing process. With ageing, the main changes regarding thyroid physiology and function are: a reduction of thyroid iodine uptake, free thyroxine and free triiodothyronine synthesis and catabolism of free thyroxine while reverse triiodothyronine increases; the level of thyroid stimulating hormone remains normal with occasionally a tendency to higher limits [53].

Thyroid status does not influence significantly the climacteric syndrome; however menopause may modify the clinical expression of some thyroid diseases, particularly the autoimmune ones. Though the thyroid function is not directly involved in the pathogenesis of menopausal complications but coronary atherosclero- 
sis and osteoporosis may be aggravated in the presence of hyperthyroidism or hypothyroidism [54].

It is of note that both menopause and thyroid disease may manifest in a similar manner. Thus, for example hot flashes, sweating, heart palpitations, insomnia, irritability, or mood swings occur in the case of hyperthyroidism, whereas weight gain, constipation, skin atrophy, hair fragility may be symptoms of hypothyroidism [55]. This may cause problems in differential diagnosis on the line vasomotor symptoms-thyroid disorders, especially when menopausal women complain of an increase in body weight, fatigue and mood swings.

In the case of hormonal treatment in women with hypothyroidism the effect of estrogen on the thyroid (an increase in thyroxine binding globulin) should be considered. However, the co-occurrence of thyroid disease should not affect the decision to initiate HT, and the treatment must be conducted according to generally accepted standards [56].

\section{Treatment}

The above mentioned menopausal disorders have a negative impact on the quality and length of women's life. Therefore, the management strategy in menopausal women should primarily focus on preventive measures, not only on pharmaceutical treatment. First of all, certain lifestyle modifications should be recommended, including (1) moderate physical activity (at least 30 minutes of moderate exercise at least three times a week, optionally in combination with two exercise sessions with load); (2) 'healthy' diet, for example, $<5$ g of salt per day (British Hypertension Society guidelines), less than $300 \mathrm{mg}$ of cholesterol/day, $1 \mathrm{~g}$ of calcium and $800 \mathrm{IU}$ of vitamin $\mathrm{D}_{3}$ per day, which could be achieved, inter alia, by reducing fat intake (including saturated fats) and carbohydrates intake (including sugars), increased consumption of fruit, vegetables and marine fish, (3) giving up smoking and alcohol, and (4) intellectual activity. Such measures should lead to body weight normalization aiming at BMI levels below $25 \mathrm{~kg} / \mathrm{m}^{2}$. Obviously, such a course of action could prove to be inadequate and additional pharmacological treatment of insulin resistance, obesity, dyslipidemia or hypertension could be required [57].

According to the current data, $\mathrm{HT}$ is not recommended as a prevention strategy for metabolic disorders in menopause. However, as part of a comprehensive strategy to prevent chronic disease after menopause, menopausal HT, particularly ET may be considered as part of the armamentarium [58].

The choice of a specific therapy should always be aligned with a particular patient and adapted to her condition and health needs, which might require consultation and further management by a range of medical professionals.

\section{Disclosure}

Authors report no conflict of interest.

\section{References}

1. Lobo RA, Davis SR, De Villiers TJ, et al. Prevention of diseases after menopause. Climacteric 2014; 17: 540-556.

2. Stachowiak G, Stetkiewicz T, Pertyński T. Cukrzyca u kobiet menopauzalnych. Prz Menopauzalny 2011; 10: 173-180.

3. Matthews KA, Crawford SL, Chae CU, et al. Are changes in cardiovascular disease risk factors in midlife women due to chronological aging or to the menopausal transition? J Am Coll Cardiol 2009; 54: 2366-2373.

4. Derby CA, Crawford SL, Pasternak RC, et al. Lipid changes during the menopause transition in relation to age and weight: the Study of Women's Health Across the Nation. Am J Epidemiol 2009; 169: 1352-1361.

5. Matthews KA, Meilahn E, Kuller LH, et al. Menopause and risk factors for coronary heart disease. N Engl J Med 1989; 321: 641-646.

6. Fan AZ, Dwyer J. Sex differences in the relation of HDL cholesterol to progression of carotid intima-media thickness: the Los Angeles Atherosclerosis Study. Atherosclerosis 2007; 195: e191-196.

7. Woodard GA, Brooks MM, Barinas-Mitchell E, et al. Lipids, menopause, and early atherosclerosis in Study of Women's Health Across the Nation Heart women. Menopause 2011; 18: 376-384.

8. Yazdani S, Sharbatdaran M, Abedi Samakoosh M, et al. Glucose tolerance and lipid profile changes after surgical menopause. Caspian J Intern Med 2014; 5: 114-117.

9. Kabir F, Jahan N, Sultana N, Akter R. Lipid profile status in surgical menopause. J Bangladesh Soc Physiol 2011; 6: 127-133.

10. Tuna V, Alkis I, Safiye As, et al. Variations in blood lipid profile, thrombotic system, arterial elasticity and psychosexual parameters in the cases of surgical and natural menopause. Aust N Z J Obstet Gynaecol 2010; 50: 194-199.

11. Xu Y, Lin J, Wang S, et al. Combined estrogen replacement therapy on metabolic control in postmenopausal women with diabetes mellitus. Kaohsiung J Med Sci 2014; 30: 350-361.

12. Clark NG, Fox KM, Grandy S; SHIELD Study Group. Symptoms of diabetes and their association with the risk and presence of diabetes: findings from the Study to Help Improve Early evaluation and management of risk factors Leading to Diabetes (SHIELD). Diabetes Care 2007; 30: 2868-2873.

13. Rossi R, Origliani G, Modena MG. Transdermal 17-beta-estradiol and risk of developing type 2 diabetes in population of healthy, nonobese postmenopausal women. Diabetes Care 2004; 27: 645-649.

14. North American Menopause Society. Estrogen and progestogen use in postmenopausal women: 2010 position statement of The North American Menopause Society. Menopause 2010; 17: 242-255.

15. Peterlik M, Cross HS. Vitamin D and calcium insufficiency-related chronic diseases: molecular and cellular pathophysiology. Eur J Clin Nutr 2009; 63: 1377-1386.

16. Kalyani RR, Franco M, Dobs AS, et al. The association of endogenous sex hormones, adiposity, and insulin resistance with incident diabetes in postmenopausal women. J Clin Endocrinol Metab 2009; 94: 4127-4135.

17. Ding EL, Song Y, Manson JE, et al. Sex hormone-binding globulin and risk of type 2 diabetes in women and men. N Engl J Med 2009; 361: 1152-1163.

18. Kanaya AM, Herrington D, Vittinghoff E, et al. Glycemic effects of postmenopausal hormone therapy: the Heart and Estrogen/progestin Replacement Study. A randomized, double-blind, placebo controlled trial. Ann Intern Med 2003; 138: 1-9.

19. Margolis KL, Bonds DE, Rodabough RJ, et al. Effect of oestrogen plus progestin on the incidence of diabetes in postmenopausal women: results from the Women's Health Initiative Hormone Trial. Diabetologia 2004; 47: 1175-1187.

20. Markopoulos MC, Rizos D, Valsamakis G, et al. Hyperandrogenism in women with polycystic ovary syndrome persists after menopause. J Clin Endocrinol Metab 2011; 96: 623-631.

21. Puurunen J, Piltonen T, Jaakkola P, et al. Adrenal androgen production capacity remains high up to menopause in women with polycystic ovary syndrome. J Clin Endocrinol Metab 2009; 94: 1973-1978. 
22. Schmidt J, Brännström M, Landin-Wilhelmsen K, Dahlgren E. Reproductive hormone levels and anthropometry in postmenopausal women with polycystic ovary syndrome (PCOS): a 21-year follow-up study of women diagnosed with PCOS around 50 years ago and their agematched controls. J Clin Endocrinol Metab 2011; 96: 2178-2185.

23. Puurunen J, Piltonen T, Morin-Papunen L, et al. Unfavorable hormonal, metabolic, and inflammatory alterations persist after menopause in women with PCOS. J Clin Endocrinol Metab 2011; 96: 1827-1834.

24. Markopoulos MC, Valsamakis G, Kouskouni E, et al. Study of carbohydrate metabolism indices and adipocytokine profile and their relationship with androgens in polycystic ovary syndrome after menopause. Eur J Endocrinol 2012; 168: 83-90.

25. Legro RS. Polycystic ovary syndrome. Long term sequelae and management. Minerva Ginecol 2002; 54: 97-114.

26. Velling Magnussen L, Mumm H, Andersen M, Glintborg D. Hemoglobin A1c as a tool for the diagnosis of type 2 diabetes in 208 premenopausal women with polycystic ovary syndrome. Fertil Steril 2011; 96: 1275-1280.

27. Hoeger K. Obesity and weight loss in polycystic ovary syndrome. Obstet Gynecol Clin North Am 2001; 28: 85-97.

28. Lambrinoudaki I. Cardiovascular risk in postmenopausal women with the polycystic ovary syndrome. Maturitas 2011; 68: 13-16.

29. McGowan MP. Polycystic ovary syndrome: a common endocrine disorder and risk factor for vascular disease. Curr Treat Options Cardiovasc Med 2011; 13: 289-301.

30. Schmidt J, Landin-Wilhelmsen K, Brännström M, Dahlgren E. Cardiovascular disease and risk factors in PCOS women of postmenopausal age: a 21-year controlled follow-up study. J Clin Endocrinol Metab 2011; 96 3794-3803.

31. Kandaraki E, Christakou C, Diamanti-Kandarakis E. Metabolic syndrome and polycystic ovary syndrome... and vice versa. Arq Bras Endocrinol Metabol 2009; 5: 227-237.

32. Shah D, Bansal S. Polycystic ovaries - beyond menopause. Climacteric 2014; 17: 109-115.

33. Amsterdam ESHRE/ASRM-Sponsored 3rd PCOS Consensus Workshop Group. Consensus on women's health aspects of polycystic ovary syndrome (PCOS). Hum Reprod 2012; 27: 14-24.

34. Pertyński T. Kobieta w wieku okołomenopauzalnym i pomenopauzalnym. Medycyna po Dyplomie 1997; 6: 13-19.

35. Toth MJ, Sites CK, Poehlman ET, Tchernof A. Effect of menopausal status on lipolysis: comparison of plasma glycerol levels in middle-aged premenopausal and early, postmenopausal women. Metabolism 2002; 51: 322-326

36. Bagnoli VR, Fonseca AM, Arie WM, et al. Metabolic disorder and obesity in 5027 Brazilian postmenopausal women. Gynecol Endocrinol 2014; 30: 717-720.

37. Waki H, Tontonoz P. Endocrine functions of adipose tissue. Annu Rev Pathol 2007: 2: 31-56.

38. Reaven G, Abbasi F, McLaughlin T. Obesity, insulin resistance, and cardiovascular disease. Recent Prog Horm Res 2004; 59: 207-223.

39. Matsuzawa Y. Therapy insight: adipocytokines in metabolic syndrome and related cardiovascular disease. Nat Clin Pract Cardiovasc Med 2006; 3: 35-42.
40. Fournet N, Judd HL. Menopause overview. In: Reproductive Medicine and Surgery. Wallach EE, Zacur HA (eds.). Mosby, St. Louis 1995; 961-981.

41. Ben Ali S, Belfki-Benali H, Aounallah-Skhiri H, et al. Menopause and metabolic syndrome in tunisian women. Biomed Res Int 2014; 2014: 457131.

42. Lobo RA. Metabolic syndrome after menopause and the role of hormones. Maturitas 2008; 60: 10-18.

43. Polotsky HN, Polotsky AJ. Metabolic implications of menopause. Semin Reprod Med 2010; 28: 426-434.

44. Eshtiaghi R, Esteghamati A, Nakhjavani M. Menopause is an independent predictor of metabolic syndrome in Iranian women. Maturitas 2010; 65: 262-266.

45. Glueck CJ, Morrison JA, Wang P, Woo JG. Early and late menarche are associated with oligomenorrhea and predict metabolic syndrome 26 years later. Metabolism 2013; 62: 1597-1606.

46. Ebrahimpour P, Fakhrzadeh $\mathrm{H}$, Heshmat R, et al. Metabolic syndrome and menopause: a population-based study. Diabetes \& Metabolic Syndrome: Clinical Research \& Reviews 2010; 4: 5-9.

47. Gaspard U. Hyperinsulinaemia, a key factor of the metabolic syndrome in postmenopausal women. Maturitas 2009; 62: 362-365.

48. Ziaei S, Mohseni H. Correlation between Hormonal Statuses and Metabolic Syndrome in Postmenopausal Women. J Family Reprod Health 2013; 7: 63-66.

49. Chedraui P, Pérez-López FR, Escobar GS, et al. Circulating leptin, resistin, adiponectin, visfatin, adipsin and ghrelin levels and insulin resistance in postmenopausal women with and without the metabolic syndrome. Maturitas 2014; 79: 86-90.

50. Chedraui P, Escobar GS, Pérez-López FR, et al. Angiogenesis, inflammation and endothelial function in postmenopausal women screened for the metabolic syndrome. Maturitas 2014; 77: 370-374.

51. Otunctemur A, Dursun M, Ozbek E, et al. Effect of Metabolic Syndrome on Sexual Function in Pre- and Postmenopausal Women. J Sex Marital Ther 2014; 13: 1-10.

52. Lobo RA. Metabolic syndrome, adipocytokines and estrogen therapy Climacteric 2005; 8 (Suppl 2): 46.

53. Mariotti S, Franceschi C, Cossarizza A, Pinchera A. The aging thyroid. Endocr Rev 1995; 16: 686-715.

54. del Ghianda S, Tonacchera M, Vitti P. Thyroid and menopause. Climacteric 2014; 17: 225-234.

55. Roberts CG, Ladenson PW. Hypothyroidism. Lancet 2004; 363: 793-803.

56. Goodman NF, Cobin RH, Ginzburg SB, et al.; American Association of Clinical Endocrinologists. American Association of Clinical Endocrinologists Medical Guidelines for Clinical Practice for the diagnosis and treatment of menopause: executive summary of recommendations. Endocr Pract 2011; 17: 949-954.

57. The Writing Group on behalf of the Workshop Consensus Group. Aging, menopause, cardiovascular disease and HRT. International Menopause Society Consensus Statement. Climacteric 2009; 12: 368-377.

58. Stachowiak G, Zając A, Pertyński T. Zespół metaboliczny u kobiet w okresie menopauzy. Prz Menopauzalny 2009; 8: 6-10. 